\title{
Détournement du savoir féminin dans les écrits journalistiques de Claude Cahun alias « $\mathrm{M}$ »
}

\author{
Alexandra Arvisais \\ Université de Montréal - Université Lille 3
}

Claude Cahun, née Lucy Schwob, a été effacée de l'histoire culturelle à la suite de sa mort en 1954, mais depuis une trentaine d'années, des études issues des domaines de la philosophie de l'esthétique, de l'histoire de l'art et, depuis peu, de la critique littéraire ont contribué à témoigner de son importance dans le paysage culturel de la première moitié du $\mathrm{XX}^{\mathrm{e}}$ siècle. Mieux connue pour ses photographies et son œuvre littéraire, Cahun a également été actrice de théâtre, activiste politique et essayiste. Dans les années 1910, elle publie ses 
premiers écrits dans le journal républicain Le Phare de la Loire ${ }^{1}$, qui appartient alors à son père. Elle s'inscrit ainsi dans la tradition journalistique familiale instaurée depuis l'achat du journal nantais par son grand-père George Schwob en 1876 et son rachat par son père, Maurice Schwob, en 18922. Son grandoncle Léon Cahun - philologue, orientaliste, écrivain et journaliste - a contribué au Phare, de même que son oncle, Marcel Schwob - poète symboliste, conteur, traducteur et journaliste -, qui y a aussi publié ses premiers textes et collaboré pendant plusieurs années. En 1913, Maurice Schwob accorde la rubrique de mode à sa fille et à sa compagne Suzanne Malherbe, alias Marcel Moore ${ }^{3}$, qui en assure les illustrations. Claude Cahun signe les chroniques, destinées à un public féminin, de la simple lettre « $M$ ». Elle investit ce sujet traditionnellement réservé aux femmes en y laissant apparaître sa propre vision du monde.

Grâce à des stratégies textuelles et rhétoriques telles que le masque, la pseudonymie, l'humour, l'ironie et le dédoublement de sens, Cahun dépasse les limites du cadre thématique et générique de la chronique de mode. Dans ces articles de jeunesse - elle n'a alors que dix-sept ans - , on sent se préciser la voix auctoriale de celle qui allait bientôt signer

1 Pour les textes publiés en revues, voir les Écrits de Claude Cahun (2002, p. 438-450).

2 Pour les informations biographiques, voir les ouvrages de François Leperlier (1992 et 2006).

3 Cahun et la peintre-graphiste Marcel Moore amorcent ensemble leurs carrières respectives. Elles travailleront en collaboration toute leur vie, l'auteure sollicitant le concours de l'artiste plasticienne pour intégrer la composante picturale à l'écriture dans l'espace d'une œuvre syncrétique. À propos de leur collaboration, consulter Andrea Oberhuber (2004) et Tirza True Latimer (2007). 
Vues et visions 4 et Hérö̈nes ${ }^{5}$. Les écrits journalistiques de « $\mathrm{M}$ » annoncent déjà certains traits de l'écriture cahunienne: la pseudonymie - masque parmi tant d'autres dans son œuvre - , l'ironie, l'emploi du genre masculin, le brouillage des genres (sexuels et littéraires), le jeu sur les apparences à travers les mascarades et le travestissement. Par son discours journalistique, Claude Cahun transforme un espace dévolu au féminin et construit son ethos d'intellectuelle moderniste. En mobilisant les moyens rhétoriques et textuels, la journaliste parvient à se dégager du sujet codifié de la mode féminine et à dépasser ainsi les lieux communs réservés aux femmes. En lieu et place du contenu traditionnel de cette chronique, elle instaure un discours atypique qui confronte les genres et les rôles sexués. En fonction de la trajectoire de Cahun dans les années de l'entre-deux-guerres, la voix de femme auteur qui perce derrière le masque de « $M$ » peut être définie par la volonté de transgresser les frontières identitaire et générique.

\section{Masque et déguisement}

Selon François Leperlier, «redécouvreur » de Claude Cahun et éditeur des Écrits, la chronique de mode du Phare de la Loire était supposée être assumée par un homme (2006, p. 38). Les articles ne comportent, de fait, aucune trace de sa véritable identité, que la jeune journaliste brouille en signant de la

\footnotetext{
${ }^{4}$ Ce recueil de poèmes en prose accompagnés d'illustrations de Marcel Moore a été publié dans Le Mercure de France le 16 mai 1914 et réédité par François Leperlier en 2002 (Cahun, 2002, p. 19-123).

5 D'abord publié dans Le Mercure de France et Le Journal littéraire en février 1925 et réédité dans Écrits (Cahun, 2002, p. 125-159), Hérö̈nes regroupe seize nouvelles sur l'imaginaire de la « nouvelle femme».
} 
lettre « M ». Le choix de ce nom de plume, s'il n'a pas été éclairé par Cahun, renvoie à plusieurs figures importantes de son entourage: Suzanne Malherbe alias Marcel Moore, Marcel Schwob, Maurice Schwob ou encore Mathilde Cahun, sa grandmère paternelle. Il ne s'agit d'ailleurs pas du seul pseudonyme de l'auteure, qui a aussi utilisé Claude Courlis, Daniel Douglas et, enfin, Claude Cahun, prénom au genre volontairement ambigu reprenant le patronyme de sa grand-mère. L'auteure s'inscrit dans une longue lignée de femmes qui ont publié sous un autre nom que le leur. Pensons à Hélisenne de Crenne, Delphine de Girardin, George Sand, Rachilde et Colette, pour n'en nommer que quelques-unes. L'usage du masque a permis à plusieurs femmes auteurs de faciliter leur entrée, longtemps limitée, dans le champ littéraire. Grâce au travestissement, les femmes auteurs peuvent façonner leur ethos discursif pour le rendre conforme aux normes de la société dans laquelle elles évoluent. Le masque pseudonymique, notamment, permet d'effacer les marques de généricité ou encore de se créer une identité factice. Chez Cahun, le masque devient un moyen de cumuler les traits identitaires, vrais ou faux, pour subvertir les rôles sexués et le concept d'une identité immuable.

Dans ses écrits journalistiques, Claude Cahun ne met pas de l'avant sa fausse identité et les textes portent peu de traces $\mathrm{du}$ travestissement textuel. L'identité sexuelle prétendue du chroniqueur n'est indiquée que par un passage (2002, p. 441) où elle se présente explicitement comme un homme et par le «vous» exclusif avec lequel elle s'adresse à ses lectrices. Le sexe de l'énonciateur demeure ambigu malgré une écriture au « je », car les articles comportent très peu de marques de genre, que ce soit à travers les pronoms ou l'accord grammatical, qui demeurent neutres. Le penchant de l'auteure pour les 
ambivalences et l'«indéfinition », déjà présent dans son écriture journalistique, prend plus d'ampleur dans le reste de son œuvre, où l'illisible et l'invisible règnent. Dans les textes publiés au Phare de la Loire, le recours à un pseudonyme neutre permet à Cahun de se parer des qualités intellectuelles plus facilement reconnues à l'homme qu'à la femme au début du XXe siècle. L'auteure met l'accent sur son savoir en combinant les sujets mondains tels que la mode et les bals, caractéristiques de la rubrique, et les marques d'érudition. Les chroniques abondent de renvois à la culture orientale, à l'étymologie, à la littérature et à plusieurs figures de l'Antiquité et des mythologies grecque et romaine. En multipliant les références savantes, Cahun dépasse le statut de chroniqueuse de mode et se compose un ethos d'auteure érudite.

Tout en s'inscrivant dans la tradition de femmes auteurs écrivant sous pseudonyme, Cahun renouvelle l'usage du masque en en faisant un moyen de subvertir les codes des rôles sexués. Ses articles sont théâtralisés et souvent écrits sous forme de saynètes dialoguées à forte charge ironique dans lesquelles elle se met en scène comme agent provocateur au sein de ses interlocutrices fictives : elle s'adresse à des femmes, des épouses et des mères. La chroniqueuse, par le biais de son personnage dans les saynètes, se présente comme une figure moderne qui bouleverse les idées reçues de ses interlocutrices sur l'image et la position des femmes dans la société. Cahun revêt parfois la persona rhétorique d'un homme pour encourager ses lectrices à changer de vêtements et d'occupation: «Moi, ça m'est éga[l.] Mettez mes pyjamas, fumez ma pipe et faites mes articles. Mais passez-moi votre kimono. Je servirai le thé, je mangerai les gâteaux, on me fera la cour... Non. Je suis généreux : Gardez votre part, Madame, c'est la meilleure.» (2002, p.441) Ce passage 
affiche son identité factice masculine pour mettre en lumière les conceptions du féminin associées au discours sur la mode. L'habillement est mis en relation avec l'occupation dans les sphères familiale et sociale selon la dichotomie habituelle qui associe l'homme à l'espace public et la femme au privé. Cahun suggère dès lors d'habiller autrement les femmes, en leur faisant porter «costumes, tailleurs, vestes et gilets» (p.439). Dans l'écriture et les autoportraits de Cahun, contemporains des chroniques dans Le Phare de la Loire, les mascarades et les déguisements ont une dimension performative pour le sujet qui se met en scène et se métamorphose continuellement. L'auteure interpelle ses interlocutrices, en multipliant les apostrophes, «vous» et «madame», pour les enjoindre à se dégager des conventions. Sous la plume cahunienne, la mode n'est qu'un prétexte à discourir d'un mélange des genres à opérer plus en profondeur: c'est une invitation à inverser les rôles, à faire entrer l'autre dans le même. Le sujet de sa chronique lui permet, de manière détournée, d'encourager les femmes à prendre une apparence hors norme, que ce soit en adoptant des modes inusitées ou en ajoutant à leur tenue des éléments réservés aux hommes : «Non, Monsieur le perruquier, je fais fi de vos conseils et n'en veux agir qu'à ma tête. Je composerai moi-même mon visage avec de la farine et du charbon.» (p.446) La voix du chroniqueur rejette les modèles féminins existants et appelle au libre arbitre des femmes à choisir leur "visage ", siège de leur identité, sa préférence allant à l'étrange, voire à l'inquiétant. Les articles de « $\mathrm{M}$ » font exploser la singularité des êtres en valorisant l'individualité par rapport à la conformité et en avançant le modèle d'une figure féminine dont l'originalité éclate sur la page, Cahun elle-même l'exemplifiant dans ses premiers autoportraits. 
Tout au long de sa carrière, l'auteure-artiste a utilisé le masque pour brouiller les identités et les genres ${ }^{6}$. Au-delà de leur aspect ludique, ces mascarades mettent en scène un moi multiple, en perpétuelle métamorphose ${ }^{7}$. L'écriture de « $M$ » manie avec humour les masques et les déguisements afin d'engendrer un sujet polymorphe: "Une question s'impose. Quelle tête peut se porter avec un habit noir?» (p. 446) L'inquiétant fait irruption dans la langue pour donner à lire et à voir un sujet indéterminé et, surtout, jamais fixe, qui peut choisir lui-même son apparence et la modifier à sa guise. Ces multiples travestissements - de nom, d'habits, de sexe tendent à montrer que l'identité n'est pas une donnée figée, mais constamment réinventée.

\section{Stratégies de détournement}

Par le truchement de la rubrique de mode, Cahun tente de faire exploser le carcan qui fige l'image et le statut de la femme au début du XXe siècle. Elle procède ainsi à un travail de dissidence que Julia Kristeva décrit comme étant un "démontage impie et impitoyable des mécanismes de discours, de pensée, d'existence » (p. 7). L'intellectuel doit maintenir une "position analytique à l'égard des identités conceptuelles, subjectives, sexuelles, linguistiques » (p. 8). À la lumière de la définition de Kristeva, on peut voir dans les écrits journalistiques de Cahun, d'abord, un procédé de démantèlement des attentes du lectorat face à la rubrique de mode. En effet, le lecteur ne peut déceler dans les articles de « $\mathrm{M} »$ des informations pratiques sur les

\footnotetext{
6 À propos des mascarades cahuniennes, voir, entre autres, Julie Hétu (2005), Charlotte Maria (2011) et Andrea Oberhuber et Nadine Schwakopf (2011).

7 Voir à ce sujet Catherine Baron (2004).
} 
dernières tendances. L'auteure repense autrement, à travers cette thématique, le masculin et le féminin en envisageant un sujet au genre "indéfini » qui emprunte à chacun des éléments de leur aspect physique, une représentation qui prendra de plus en plus d'importance dans l'œuvre cahunienne. Par l'ironie et le dédoublement de sens, Cahun substitue à la question de la mode féminine la représentation d'un nouveau modèle de femme moderne. De fait, elle détourne les thèmes de la rubrique de mode au profit d'un discours sur l'apparence, les mœurs et la place de la femme dans la société : «Ne savez-vous donc pas que pour les uns la mode est un grand art et pour d'autres un prétexte à philosopher? » (Cahun, 2002, p. 441) La chroniqueuse suggère à ses lectrices de rejeter les normes du bon goût pour célébrer leur individualité. De cette façon, elle aborde le statut de la femme au tournant du siècle qui, malgré les changements amorcés, reste encore celui d' «Ange du foyer», pour reprendre l'expression de Virginia Woolf, selon laquelle la femme ne peut écrire avant d'avoir tué le spectre de l'ange du foyer (p. 28). Par l'accumulation de stratégies textuelles et rhétoriques pour démonter le contenu codifié de la chronique, Cahun traverse la frontière entre l'ange du foyer et l'intellectuelle. Sous la plume de « $\mathrm{M}$ », le sujet vestimentaire dérive vers une analyse de l'écart entre l'extérieur et l'intérieur. Elle établit un parallèle entre l'univers domestique dans lequel la femme est enfermée et les vêtements qu'elle porte. Dénonçant les effets de mode qui briment l'originalité de chacune, Cahun affirme que l'habit doit refléter l'esprit (2002, p. 447). Elle suggère dès lors de s'habiller simplement, car les vêtements féminins - trop serrés, trop étroits - entravent le libre esprit : "Dans cette longue chemise aux plis droits, vous serez à votre gré Cléopâtre, Iphigénie, toutes les héroïnes, voire même vous. » (p. 440) Cahun associe la liberté 
du corps au développement des aspirations intellectuelles et sociales des femmes. Les figures de Cléopâtre, d'Iphigénie et d'Ève sont convoquées pour exemplifier les possibles qui s'offrent à elles et encourager leur émancipation. La même année, dans son recueil de nouvelles Héroïnes, l'auteure reprend ce procédé et réécrit l'histoire de plusieurs personnages féminins littéraires, bibliques ou mythiques en la confrontant à la $\mathrm{New}$ $W_{o m a n}^{8}$ qui émerge dans l'entre-deux-guerres.

Cahun cumule les stratégies de détournement antiphrase, ironie et dédoublement de sens - mises au service d'un imaginaire de la nouvelle femme - émancipée, libérée des lieux communs attribués au masculin et au féminin (notamment des tâches ménagères et des codes vestimentaires) - qu'elle crée à travers ses chroniques. D'abord, grâce au dédoublement de sens, la vision du monde de Cahun apparaît dans ses articles tout en demeurant dans les limites thématiques de la rubrique de mode. À titre d'exemple, sous couvert de parler d'élégance, elle dénonce le peu de latitude laissée à la femme dans la société et suggère à celle-ci de prendre l'uniforme masculin pour $\mathrm{y}$ remédier :

Si vous êtes élégante, le bas de votre robe contribue à l'ensemble et passe inaperçu. Vous ne devez donc sous aucun prétexte vous laisser entraver: l'embarras de votre démarche attirera l'attention. Exigez la liberté de vos pas : le tailleur est l'uniforme du footing. Fendez-le par devant, vous êtes à l'aise et cela ne fait pas un pli; ou bien, sur chaque couture de côté, ménagez un pli fermé qui s'ouvre pendant la marche. [...] Eh bien! Madame, vous me croirez, si vous voulez, c'est la déformation des modes masculines qui féminise vos tailleurs. (2002, p. 439)

8 Pour la figure de la New Woman ou de la nouvelle femme, consulter Sally Ledger (1997), Angelique Richardson et Chris Willis (dir.) (2002 [2001]) et Ann L. Ardis (1990). 
Cahun s'insurge contre le lieu commun selon lequel les femmes doivent se fondre dans la masse et ne peuvent afficher leur singularité. L'obligation d'être élégante lui semble une manière de contenir l'élan de la femme et de masquer sa vraie nature, métaphorisée par le pas freiné par le vêtement. L'habillement modifié, l'attitude même de la femme est appelée à se transformer. Cahun propose en contrepartie de s'approprier la mode masculine comme un complément au féminin. Ses écrits journalistiques témoignent des changements de l'image de la femme observables durant ces années, particulièrement à Paris : apparaissent effectivement les silhouettes androgynes, de même que les figures de la garçonne et de l'amazone' ${ }^{9}$. Figure à contre-courant, Cahun reproche de suivre bêtement la mode et encourage les femmes à trancher de l'ensemble, par le truchement d'un gilet en soie brochée (p. 439). Les articles dans Le Phare de la Loire attaquent les normes sociales qui ont trait à l'image de la femme. Le détournement suivant du célèbre passage d'Hamlet montre éloquemment la logique binaire dans laquelle chaque sexe est coincé, l'un se voyant associé aux domaines de l'esprit, l'autre au corps : «Être ou paraître, voilà la question. » (p. 440) En reprenant la phrase de Shakespeare, Cahun dévoile le peu d'options offertes aux femmes. Elle manipule les citations, les adages, les clichés, une stratégie textuelle qui est omniprésente dans son œuvre et qui atteint son paroxysme dans le livre hybride Aveux non avenus. La contestation de l'exclusion des femmes des domaines du savoir et leur relégation à l'espace du corps passe également par la langue empreinte d'ironie de Cahun. La jeune journaliste prend part aux débats de son époque par sa prose mordante

9 Voir Whitney Chadwick et Tirza True Latimer (2003) et Christine Bard (1998). 
résolument ancrée dans la modernité : «Figurez-vous qu'on voudrait lancer à Paris les pyjamas pour dame. Êtes-vous féministe? » (p. 440) Cette réplique cinglante rend compte de la réaction des contemporains de Cahun aux modifications dans l'apparence et l'attitude des femmes, tout comme elle laisse entendre, par l'antiphrase, sa propre pensée. Si, portée par une voix censée être masculine, l'ironie présente toutefois le danger d'être comprise au premier degré par ses lectrices, l'approche $\mathrm{du}$ thème de la mode par « $\mathrm{M}$ » dans l'ensemble de ses chroniques permet de déceler le double sens dans son propos. Effectivement, le discours qui supplée au savoir dit féminin de la mode et de la domesticité propose aux femmes de se détourner des anciens codes de la féminité pour faire siennes les marques du genre masculin, notamment une silhouette androgyne, une part active dans la société et l'érudition. Ses références savantes et la distance critique prise à travers les stratégies de détournement érigent une figure d'intellectuelle qui confronte les idées reçues en montrant que la femme peut déborder du savoir ménager et vestimentaire.

\section{Construction d'une voix auctoriale}

Dans le propos journalistique de " $\mathrm{M}$ », on sent se mettre en place la voix d'auteure de celle qui deviendra Claude Cahun. De la lecture des articles ressortent certains invariants de son écriture et de sa pratique photographique. Notons d'abord le rejet de la dichotomie entre le masculin et le féminin, illustré par le bouleversement des caractéristiques physiques attribuées à chaque sexe, ainsi que l'appropriation des savoirs associés au masculin. L'auteure recourt aux stratégies textuelles 
et rhétoriques du masque et du détournement de sens - que l'on retrouve au cœur d'Hérö̈nes et d'Aveux non avenus, de même que dans les autoportraits et les photomontages - pour adapter la rubrique de mode à ses thèmes de prédilection. Les articles de « $\mathrm{M}$ » témoignent déjà de son plaisir à jouer avec la polysémie de l'image et du langage. En endossant le double statut de journaliste et d'écrivain, à l'instar de plusieurs intellectuels des XIX ${ }^{\mathrm{e}}$ et $\mathrm{XX}^{\mathrm{e}}$ siècles, Cahun développe aussi son goût pour la prose essayistique, qui ressurgira plus tard dans l'essai poétique Les Paris sont ouverts ${ }^{10}$ et les pamphlets du Soldat Ohne Namen durant la Seconde Guerre mondiale.

Par ailleurs, dès ses premiers écrits, grâce au cadre générique de la chronique de mode qui inclut fréquemment des illustrations, Cahun pratique la collaboration interartistique avec sa partenaire Marcel Moore. La peintre-graphiste effectue les dessins de patrons, de modèles ou de mannequins qu'il est d'usage d'inclure dans une telle rubrique. Elle incorpore à ses modèles des éléments masculins : mannequins au visage dur et aux traits androgynes et veston pour homme noir juxtaposé aux tenues habituelles. L'esthétique du couple d'auteure-artiste est également affinée dans le discours journalistique qui repousse les effets de mode au profit de la singularité. Dans une de ses chroniques, Cahun reproche aux peintres et à son propre journal de succomber à ce qu'elle appelle la «mode de la couleur»: "Alors, on sacrifie la forme à la couleur; la femme n'a plus la ligne, le dessin plus de traits [...]. Mais la mode est là, elle ne supporte guère qu'on discute avec elle des goûts et des couleurs. » (2002, p. 448) Par cette sortie, Cahun façonne sa propre pratique du dessin, telle qu'elle et Moore la mettent en

10 Publié en 1934, cet essai a été réédité dans Écrits (2002). 
œuvre la même année dans Vues et visions, recueil de poèmes en prose dans lequel sont disposées des esquisses en noir et blanc aux traits foncés. Si les photomontages procèdent par collage et superposition de fragments iconiques, le dessin demeure généralement plus simple dans son œuvre. Les nouvelles d'Hérö̈nes et les fragments textuels d'Aveux non avenus sont séparés par divers symboles à la signification obscure (formes géométriques, symboles typographiques, cœur, bouche, œil). Les dessins incorporés à la chronique de mode sont toutefois encore très éloignés du travail du texte et de l'image que feront plus tard les collaboratrices et qui ne tiendra plus d'aucune façon du rapport illustratif d'un média par l'autre.

Ses écrits dans Le Phare de la Loire établissent quelques grandes lignes de la poétique d'auteure de Claude Cahun et la positionnent dans l'entre-deux qui lui sera toujours cher11. La forme des chroniques, qui emprunte à la rubrique de mode, au théâtre et à l'essai, de même que les questionnements sur l'androgynie, le genre et les rôles sexués permettent de parler, chez Cahun, d'une propension à transgresser, sans jamais se situer ni de l'un ni de l'autre côté de la frontière que caractérise le sujet moderniste mis en mots à travers son écriture journalistique.

\section{Claude Cahun alias « M », intellectuelle dissidente}

Michelle Perrot rappelle la difficulté pour les femmes d'acquérir le titre d'intellectuelle, souvent défini non seulement par l'œuvre, mais aussi par l'implication sociale : «on entend par

\footnotetext{
11 Consulter Andrea Oberhuber (2007).
} 
"intellectuel" "un homme ou une femme qui applique à l'ordre politique une notoriété acquise ailleurs", la plupart du temps par l'écriture, les sciences ou les arts» (p.101). Même si plusieurs femmes ont pris la plume du XVIe au XIXe siècles, elles ont été confinées à des genres dits féminins, tels que le roman, la correspondance et le journal intime, qui rendent difficile leur reconnaissance en tant qu'intellectuelles. Le début du XXe siècle marque un tournant majeur pour les écrivaines, qui voient l'espace culturel et socio-économique s'ouvrir devant elles, notamment grâce à la mobilisation des intellectuels autour de l'affaire Dreyfus, de l'accès aux études et au marché du travail et de l'essor du féminisme qui en découle (Perrot, p.110). La littérature fait écho à cette ouverture par l'apparition des figures de la New Woman et de la "femme non liée ${ }^{12}$ ». La volonté de Claude Cahun de s'inscrire comme intellectuelle érudite et marginale transparaît à travers le discours moderniste à portée philosophique et sociale qui sous-tend ses écrits journalistiques. Même si l'espace qui lui est dévolu, celui de la chronique de mode, demeure restreint, elle l'investit d'une parole novatrice qui appelle les femmes, via un bouleversement des codes vestimentaires genrés, à sortir du moule et à refuser les rôles prédéterminés qu'on leur attribue. Par les stratégies du masque, de l'ironie et du dédoublement de sens, Claude

12 Nathalie Heinich étudie la figure de la femme «non liée» et précise l'exemplification qu'en font les femmes auteurs : «De Germaine de Staël à George Sand, de Colette à Virginia Woolf ou à Simone de Beauvoir, les femmes écrivains sont doublement emblématiques de la femme libre telle qu'elle s'affirmera à l'époque moderne. Car non contentes de travailler et de gagner leur vie par elles-mêmes, sans dépendre d'un homme, elles sont en mesure de construire par l'écriture des représentations durables et largement diffusables de ce qu'elles sont ou veulent être : en écrivant, elles proposent des figurations romanesques de leur propre position, et en signant, elles affirment publiquement leur activité, leur identité d'écrivain. » (2004, p. 147) 
Cahun parvient à transformer le savoir de la mode et de l'univers domestique que la presse réservait alors à la femme. Sa trajectoire, dont j'ai voulu mettre en évidence quelques grands axes qui se trouvent en germe dans ses articles pour Le Phare de la Loire, fait ressortir une figure d'auteure qui transgresse les frontières générique, sexuelle, identitaire et esthétique. Cahun s'inscrit dans la définition que donne Kristeva de la fonction de l'intellectuel dissident :

Dire la singularité des inconscients, des désirs, des besoins. Mettre en jeu les identités et / ou les langages des individus et des groupes. Devenir l'analyste des ensembles sociaux comme impossibles: des discours homogènes et des institutions consacrées comme impossibles. S'affirmer comme révélateur de l'Impossible. (p. 4)

En tant que femme, juive et lesbienne, Claude Cahun a certes expérimenté les états limites de l'identité et les a transcrits dans son cuvre déroutante et parfois obscure. Dans ses chroniques, l'auteure encourage ses lectrices à se définir hors des sentiers balisés du masculin et du féminin qui régissent à la fois l'habillement et la fonction sociale. Il s'agit justement de la position adoptée par le sujet cahunien qui, entre les mots et les images, cherche constamment à se réinventer à travers les mascarades et les travestissements textuels et photographiques. À la singularité du sujet, fait écho l'originalité de son œuvre protéiforme qui se place sous le signe de l'hybridité tant générique que médiatique. Les chroniques de « $\mathrm{M}$ » jettent les bases de l'œuvre à venir en déplaçant les limites génériques et thématiques de la rubrique de mode pour revendiquer le droit à la subjectivité et à la singularité qui émerge des représentations plurielles du sujet. 


\section{Bibliographie}

ARDIS, Ann L. (1990), New Women, New Novels. Feminism and Early Modernism, Londres, Rutgers University Press.

BARD, Christine. (1998), La Garçonne, Paris, Flammarion.

BARON, Catherine. (2004), Métamorphose et écriture autobiographique dans Aveux non avenus de Claude Cahun, Université de Montréal, mémoire de maîtrise présenté au Département des littératures de langue française.

CaHun, Claude. (2011 [1930]), Aveux non avenus, Paris, Mille et une nuits.

-. (2002), Écrits, édition présentée et établie par François Leperlier, Paris, Jean-Michel Place.

CHADWICK, Whitney et Tirza True Latimer. (2003), The Modern Woman Revisited. Paris Between the Wars, Londres, Rutgers University Press.

KRISTEVA, Julia. (1977), «Un nouveau type d'intellectuel : le dissident », Tel Quel, no 74, p. 3-8.

HEINICH, Nathalie. (2004), «Femmes écrivains: écriture et indépendance », dans Nicole Racine et Michel Trebitsch (dir.), Intellectuelles. Du genre en histoire des intellectuels, Bruxelles, Complexe, coll. « Histoire du temps présent », p. 137-155.

HÉTU, Julie. (2005), Le Motif du masque dans l'œuvre littéraire et photographique de Claude Cahun, Université de Montréal, mémoire de maîtrise présenté au Département des littératures de langue française. 
LEDGER, Sally. (1997), The New Woman. Fiction and Feminism at the fin de siècle, Manchester et New Work, Manchester University Press.

LEPERLIER, François. (1992), Claude Cahun: l'écart et la métamorphose, Paris, Jean-Michel Place.

—. (2006), Claude Cahun. L'exotisme intérieur, Paris, Fayard.

MARIA, Charlotte. (2011), "Claude Cahun ou les masques de l'identité », dans Jean-Philippe Beaulieu et Andrea Oberhuber (dir.), Jeu de masques. Les femmes et le travestissement textuel (1500-1940), Saint-Étienne, Presses de l'Université de Saint-Étienne, coll. «L'école du genre», p. 227-237.

OBERHUBER, Andrea. (2004), «Aimer, s'aimer à s'y perdre? Les jeux spéculaires de Cahun-Moore », Intermédialités, no 4, p. 87-114.

-. (2007), Claude Cahun : contexte, posture, filiation. Pour une esthétique de l'entre-deux, Montréal, coll. « Paragraphes ».

- et Nadine Schwakopf. (2011), " Masques et travestissement du sujet féminin dans l'œuvre autographique de Claude Cahun », dans Jean-Philippe Beaulieu et Andrea Oberhuber (dir.), Jeu de masques. Les femmes et le travestissement textuel (1500-1940), Saint-Étienne, Presses de l'Université de SaintÉtienne, coll. «L'école du genre », p. 239-257.

PERROT, Michelle. (2004), «Les intellectuelles dans les limbes du XIXe siècle », dans Nicole Racine et Michel Trebitsch (dir.), Intellectuelles. Du genre en histoire des intellectuels, Bruxelles, Complexe, coll. «Histoire du temps présent», p. 101-114. 
RiCHARDSON, Angelique et Chris Willis (dir.). (2002 [2001]), The New Woman in Fiction and in Fact. Fin-de-siècle Feminisms, Hampshire et New York, Palgrave Macmillan.

True Latimer, Tirza. (2007), «Entre Claude Cahun et Marcel Moore ", dans Andrea Oberhuber, Claude Cahun: contexte, posture, filiation. Pour une esthétique de l'entre-deux, Montréal, coll. « Paragraphes », p. 31-42.

Woolf, Virginia. (1983), Les Fruits étranges et brillants de l'art, Paris, Des femmes.

\section{Résumé}

Dans les années 1910, Claude Cahun publie ses premiers écrits journalistiques dans Le Phare de la Loire. Elle y tient la rubrique de mode, qu'elle signe de la simple lettre « $\mathrm{M}$ ». Cahun investit ce sujet traditionnellement destiné aux femmes en y laissant apparaître sa propre vision du monde. Pour transformer un espace dévolu au féminin, tel que la mode, et en dépasser les lieux communs, l'auteure recourt aux stratégies textuelles et rhétoriques du masque et du dédoublement de sens. À travers l'usage de la pseudonymie, de l'ironie, de la confusion des genres sexuels et littéraires et des mascarades, on sent se préciser la voix auctoriale de Cahun.

\section{Abstract}

In the 1910s, Claude Cahun publishes her first journalistic writings in Le Phare de la Loire. She writes the fashion column which she signs only « $\mathrm{M}$ ». Cahun takes on this subject traditionally intended for women and lets her own worldview transpire. To transform this space of feminity and transcend the fashion column's limited thematic and generic framework, the writer uses textual and rhetorical strategies. Through pseudonymity, irony, confusion amongst gender and literary genre and masquerade, Cahun's authorial voice takes shape. 